\title{
No Effect of High Fat Diet-Induced Obesity on Spontaneous Reporter Gene Mutations in gpt Delta Mice
}

\author{
Shinji Takasu ${ }^{1}$, Yuji Ishii ${ }^{1}$, Kohei Matsushita ${ }^{1}$, Ken Kuroda ${ }^{1}$, Aki Kijima ${ }^{1}$, Yukio \\ Kodama $^{2}$, Kumiko Ogawa ${ }^{1}$, Takashi Umemura ${ }^{1 *}$
}

\begin{abstract}
A large number of epidemiological studies have demonstrated that obesity is a risk factor for several human cancers. Several animal studies using rodents with diet-induced or genetic obesity have also demonstrated that obesity can promote tumor development. However, the effects of obesity on the early stages of carcinogenesis, and especially on the spontaneous occurrence of somatic gene mutations, remain unclear. To investigate the effects of obesity on the rate of spontaneous gene mutations, we performed reporter gene mutation assays in liver, kidney, and colon, organs in which obesity appears to be associated with cancer development on the basis of epidemiological or animal studies, in mice with high fat diet (HFD)-induced obesity. Six-week-old male and female C57BL/6 gpt delta mice were fed HFD or standard diet (STD) for 13 or 26 weeks. At the end of the experiments, reporter gene mutation assays of liver, kidney, and colon were performed. Final body weights and serum leptin levels of male and female mice fed HFD for 13 or 26 weeks were significantly increased compared with corresponding STD-fed groups. Reporter gene mutation assays of liver, kidney, and colon revealed that there were no significant differences in gpt or Spi- mutant frequencies between STD- and HFD-fed mice in either the 13-week or 26-week groups. These results indicate that HFD treatment and consequent obesity does not appear to influence the spontaneous occurrence of somatic gene mutations.
\end{abstract}

Keywords: Obesity - in vivo mutagenicity

Asian Pac J Cancer Prev, 15 (17), 7149-7152

\section{Introduction}

A large number of epidemiological studies have demonstrated that obesity is a risk factor for several human cancers. A previous systematic review and meta-analysis showed that a $5 \mathrm{~kg} / \mathrm{m}^{2}$ increase in body mass index (BMI), commonly used as a marker of body fatness, is strongly associated with increased risk of several human cancers including colon [risk ratio (RR) 1.24] and renal cancers (RR 1.24) in men, and renal cancer (RR 1.34) in women (Renehan et al., 2008). A greater BMI is also a modifiable risk factor for colon cancer for Asia population (Morrison et al., 2013). On the basis of epidemiological studies, the World Cancer Research Fund and the American Institute for Cancer Research suggested that greater body fat is associated with increased risk for several cancers, including colorectal, kidney, pancreas, breast (postmenopausal) and endometrial cancer, and has been suspected as a causative factor for gallbladder cancer. In addition, there is limited evidence suggesting that greater body fatness increases the risk of liver cancer (World Cancer Research Fund, 2007).

Several animal studies using rodents with diet- induced or genetic obesity have also indicated that obesity has the potential to promote tumor development. Excessive feeding of dietary fat such as corn oil has been reported to promote azoxymethane (AOM)-induced colon carcinogenesis in F344 rats (Reddy and Maeura, 1984). The $d b / d b$ mouse, a genetically altered animal model with obesity due to a functional defect in the long-form leptin receptor (Lee et al., 1996), is sensitive to chemical carcinogenesis such as AOM-induced colon carcinogenesis (Hirose et al., 2004) and diethylnitrosamine (DEN)-induced liver carcinogenesis (Iwasa et al., 2010). In previous studies, various pathophysiological mechanisms linking obesity to cancer have been suggested. Insulin resistance and subsequent abnormal activation of insulin-like growth factor (IGF) and the IGF-1 axis has been considered to play a key role in carcinogenesis (van Kruijsdijk et al., 2009; Shimizu et al., 2011). Other possible mechanisms include dysfunction of adipose tissue due to dysregulation of adipocytokines such as adiponectin, leptin, and plasminogen activator inhibitor-1 resulting from the enlargement of adipocytes (Shimizu et al., 2011). Thus, the effects of obesity on the promotion of tumor development and the associated cancer cell biology 
have been widely investigated. Nevertheless, the effects of obesity on the early stages of carcinogenesis, and especially the spontaneous occurrence of somatic gene mutations, remain unclear.

Animals carrying reporter genes, such as gpt delta transgenic animals, have been utilized to estimate the in vivo mutagenicity of environmental chemicals (Nohmi et al., 2000). In addition, these animals have also been used to investigate spontaneous gene mutations affected by specific genetic defects such as p53 or Nrf2 knockout (Aoki et al., 2007; Masumura et al., 2011). In the present study, to determine the effects of obesity on spontaneous gene mutations, we performed reporter gene mutation assays of liver, kidney, and colon, which are organs in which obesity has been associated with cancer development on the basis of epidemiological or animal studies, in high fat diet (HFD)-fed obese mice.

\section{Materials and Methods}

\section{Animals}

Five-week-old male and female C57BL/6 gpt delta mice carrying 80 tandem copies of the transgene lambda EG10 per haploid genome were randomized by body weight into 2 groups. They were housed in polycarbonate cages with hardwood chips for bedding in a conventional animal facility, air-conditioned to $23 \pm 2^{\circ} \mathrm{C}$ and $55 \pm 5 \%$ humidity, on a 12 hour light-dark cycle. The protocol for this study was approved by the Animal Care and Utilization Committee of the National Institute of Health Sciences (Tokyo, Japan).

\section{Animal treatments}

Following 1 week of acclimatization, 6-week-old male and female C57BL/6 gpt delta mice were fed HFD (Quick fat diet, crude fat; $13.6 \%$ [CLEA Japan, Tokyo, Japan]) or standard diet (STD) (CE-2 diet, crude fat; 4.3\% [CLEA Japan]) ad libitum for 13 or 26 weeks. Body weights were measured once a week. At the end of the experiments, all animals were euthanized under deep anesthesia. Livers, kidneys, and colon mucosa were collected and frozen immediately in liquid nitrogen and stored at $-80^{\circ} \mathrm{C}$ for in vivo mutation assays. A portion of each of the harvested livers was fixed in 10\% neutral-buffered formalin. Fixed tissues were embedded in paraffin, sectioned, and stained with hematoxylin and eosin. At necropsy, blood samples were collected from the abdominal aorta for analysis of serum leptin levels using the mouse leptin Quantikine ELISA Kit (R\&D systems, MN).

\section{In vivo mutation assays}

6-Thioguanine (6-TG) and $\mathrm{Spi}^{-}$selection were performed as previously reported by Nohmi T. et al. (Nohmi et al, 2000). Briefly, genomic DNA was extracted from liver, kidney, and colon mucosa and lambda EG10 DNA was rescued as phages by in vitro packaging. For 6-TG selection, packaged phages were incubated with molten soft agar and poured onto agar plates containing chloramphenicol and 6-TG. To determine the total number of rescued plasmids, infected cells were also poured onto plates containing chloramphenicol without 6-TG. The plates were then incubated at $37^{\circ} \mathrm{C}$. Positively selected colonies were counted on day 3 and collected on day 4 . The gpt mutant frequencies (MFs) were calculated by dividing the number of gpt mutants by the number of rescued phages.

For Spi- selection, packaged phages were incubated with E. coli XL1-Blue MRA for survival titration and E. coli XL1-Blue MRAP2 for mutant selection. Infected cells were mixed with molten lambda-trypticase soft agar and poured onto lambda-trypticase agar plates. On the next day, plaques (Spi- candidates) were punched out with sterilized glass pipettes and the agar plugs suspended in SM buffer. The Spi phenotype was confirmed by spotting the suspensions on three types of plates on which XL1Blue MRA, XL1-Blue MRA P2, or WL95 P2 strains were spread with soft agar. Spi- mutants, which produced clear plaques on every plate, were counted.

\section{Statistical analysis}

All results were expressed as mean \pm SD. The data for body weights, gpt and $\mathrm{Spi}^{-} \mathrm{MFs}$, and serum leptin levels were analyzed by Student's test.

\section{Results}

\section{Obesity development}

Body weight curves are shown in Figure 1. Final body weights of male mice fed STD or HFD for 13 weeks were $30.6 \pm 1.9 \mathrm{~g}$ and $36.2 \pm 3.2 \mathrm{~g}$, and those of mice fed the diets for 26 weeks were $35.5 \pm 3.7 \mathrm{~g}$ and $45.2 \pm 3.4 \mathrm{~g}$, respectively. Final body weights of female mice fed STD or HFD for 13 weeks were $23.2 \pm 1.1 \mathrm{~g}$ and $25.7 \pm 1.9 \mathrm{~g}$, and those of mice fed the diets for 26 weeks were $26.0 \pm 2.0 \mathrm{~g}$ and $35.6 \pm 5.3 \mathrm{~g}$, respectively. Final body weights of male and female mice fed HFD for 13 or 26 weeks were significantly

$\mathbf{A}$
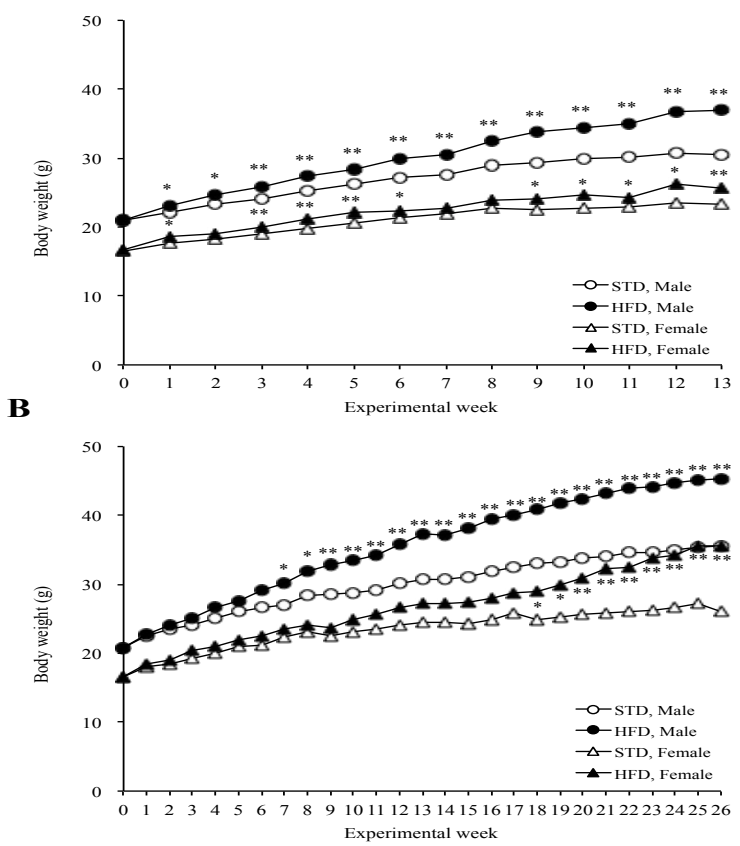

Figure 1. Body Weight Curves for C57BL/6 gpt Delta Mice Fed HFD for 13 A) or 26 B) Weeks. *,**, Significantly different from the STD Group at $p<0.05$ and $p<0.01$, respectively 


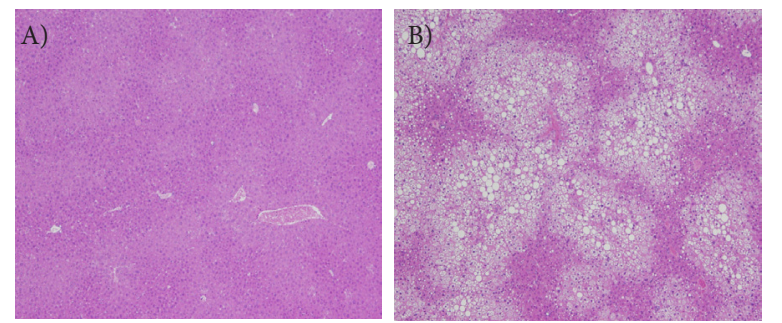

Figure 2. Histopathological Features of the Livers of C57BL/6 gpt Delta Mice Fed STD A) or HFD B) for 26 Weeks. HE Stain

A

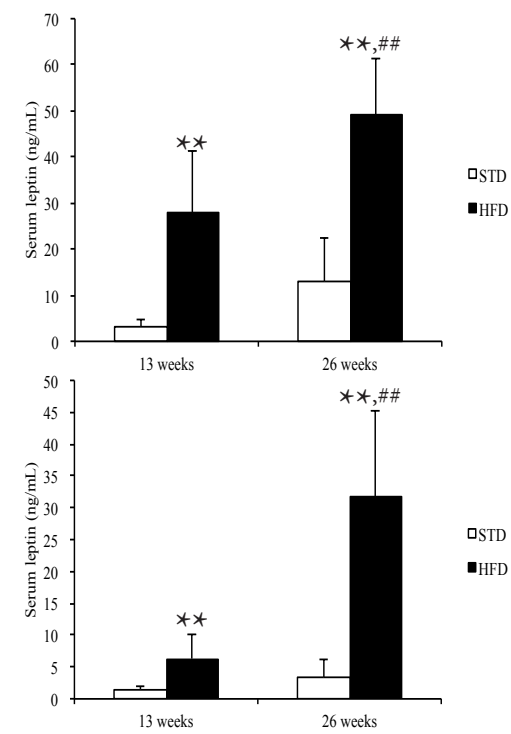

Figure 3. Serum Leptin Concentrations of Male (A) and Female (B) C57BL/6 gpt Delta Mice Fed HFD for 13 or 26 weeks. **Significantly different from the STD group at $p<0.01$.\#\#, significantly different from the group fed HFD for 13 weeks at $p<0.01$. Values are means \pm SD

Table 1. Gpt Mutant Frequencies in the Liver, Kidney and Colon of Mice Treated with STD or HFD

\begin{tabular}{lllccc}
\hline Organ sex & \multicolumn{4}{c}{ Mutant frequency $\left(\times 10^{-5}\right)^{\mathrm{a}}$} \\
& & \multicolumn{2}{c}{13 weeks } & \multicolumn{2}{c}{26 weeks } \\
\hline \multirow{2}{*}{ Liver } & Male & $0.24 \pm 0.13^{\mathrm{b}}$ & $0.22 \pm 0.15$ & $0.73 \pm 0.11$ & $0.61 \pm 0.17$ \\
& Female & $0.72 \pm 0.24$ & $0.45 \pm 0.19$ & $0.57 \pm 0.19$ & $0.52 \pm 0.21$ \\
\multirow{4}{*}{ Kidney } & Male & $0.27 \pm 0.17$ & $0.36 \pm 0.28$ & $0.67 \pm 0.59$ & $0.49 \pm 0.26$ \\
& Female & $0.27 \pm 0.12$ & $0.22 \pm 0.11$ & $0.48 \pm 0.32$ & $0.36 \pm 0.21$ \\
Colon & Male & $0.38 \pm 0.12$ & $0.51 \pm 0.19$ & $0.95 \pm 0.64$ & $0.92 \pm 0.31$ \\
& Female & $0.50 \pm 0.19$ & $0.63 \pm 0.31$ & $1.10 \pm 0.52$ & $0.80 \pm 0.42$ \\
\hline
\end{tabular}

${ }^{a}$ Mutant frequency were calculated by dividing the number of chloramphenicol and 6-TG resistant clonies by the number of chloramphenicol resistant clonies. ${ }^{\mathrm{b}} \mathrm{Mean} \pm \mathrm{SD}$

increased compared with corresponding STD-fed groups. Histopathological examination showed that the livers of mice fed HFD showed substantial steatosis (Figure 2). In addition, the final body weights of male and female mice fed STD or HFD for 26 weeks were also significantly increased compared with those of mice fed STD or HFD for 13 weeks. Serum leptin levels of male and female mice fed HFD for 13 or 26 weeks were significantly increased compared with corresponding controls (Figure 3). In addition, animals that underwent HFD feeding for 26 weeks showed significantly increased serum leptin levels compared with those of animals fed HFD for 13 weeks.
Table 2. Spi- Mutant Frequencies in rhe Liver, Kidney and Colon of Mice Treated with STD or HFD

\begin{tabular}{lllccc}
\hline Organ sex & \multicolumn{4}{c}{ Mutant frequency $\left(\times 10^{-5}\right)^{\mathrm{a}}$} \\
& & 13 weeks & \multicolumn{2}{c}{26 weeks } \\
& & STD & HFD & STD & HFD \\
\hline \multirow{2}{*}{ Liver } & Male & $0.15 \pm 0.09^{\mathrm{b}}$ & $0.19 \pm 0.08$ & $0.37 \pm 0.29$ & $0.14 \pm 0.04$ \\
& Female & $0.16 \pm 0.07$ & $0.12 \pm 0.04$ & $0.50 \pm 0.35$ & $0.23 \pm 0.08$ \\
Kidney & Male & $0.17 \pm 0.07$ & $0.31 \pm 0.23$ & $0.16 \pm 0.05$ & $0.30 \pm 0.13$ \\
& Female & $0.16 \pm 0.05$ & $0.19 \pm 0.09$ & $0.29 \pm 0.15$ & $0.19 \pm 0.01$ \\
Colon & Male & $0.20 \pm 0.16$ & $0.15 \pm 0.04$ & $0.28 \pm 0.11$ & $0.23 \pm 0.09$ \\
& Female & $0.35 \pm 0.17$ & $0.21 \pm 0.11$ & $0.31 \pm 0.30$ & $0.34 \pm 0.11$ \\
\hline
\end{tabular}

${ }^{a}$ Mutant frequency were calculated by dividing the number of plaques within WL95 (P2) by the number of plaues within XL-1 Blue MRA; ${ }^{\text {Mean } \pm \text { SD }}$

gpt and Spi- MFs in liver, kidney, and colon

Data for $g p t$ and Spi- MFs in liver, kidney, and colon from mice fed HFD for 13 or 26 weeks are summarized in Table 1 and 2. There were no significant differences in $g p t$ and Spi ${ }^{-}$MFs between the STD- and HFD-treated mice for either duration of feeding.

\section{Discussion}

In the present study, body weights of mice fed HFD were significantly higher than those of mice fed STD. The degree of body weight gain was increased with HFD feeding duration. These results imply that HFD feeding is responsible for the observed increase in body weight. In addition to the increase in body weights, histopathological analysis revealed that the livers of mice fed HFD exhibited steatosis, indicating that the mice fed HFD under the present experimental conditions became obese due to a metabolic disorder.

In recent years, a strong association between obesity and tumor development has come to be widely accepted. Some studies using rodents have suggested that obesity has the potential to promote tumor development (Hirose et al., 2004; Iwasa et al., 2010; Padidar et al., 2012). Genetically altered obese animal models such as $d b / d b$ mice, which show hepatic steatosis accompanied by obesity (Iwasa et al., 2010; Trak-Smayra et al., 2011), are reported to have a high susceptibility to chemical carcinogenesis of the liver and colon (Hirose et al., 2004; Iwasa et al., 2010).

Meanwhile, since some adipocytokines such as leptin and adiponectin have been reported to affect metabolism, inflammation, angiogenesis, and cell-cycle regulation (Vansaun, 2013), breakdown of their homeostasis due to obesity has been proposed to play an important role in the promotion of tumor development (van Kruijsdijk et al., 2009). Indeed, one of the above-mentioned studies documenting the effects of obesity on the development of colon preneoplastic lesions reported that serum leptin concentrations of mice fed a high fat diet $(60 \%$ fat by energy) were 4.2 times higher than those of mice fed a low-fat diet (10\% fat by energy) (Padidar et al., 2012). Leptin, which is a $16 \mathrm{kD}$ bioactive protein mainly secreted from adipocytes, acts as a regulator of energy balance to regulate satiety and modulate glucose and insulin homeostasis (Bjorbaek and Kahn, 2004). Leptin elicits activation of Janus-activated kinase 2 and subsequent phosphorylation of insulin receptor substrates, and subsequently initiates activation of phosphoinositide 
Shinji Takasu et al

3-kinase (PI3K)/Akt pathways through the short-form leptin receptor (Ceddia, 2005). In addition, leptin binding to the long-form leptin receptor activates signal transducer and activator of transcription (STAT) 3 and STAT5 (Ceddia, 2005). Activation of these pathways leads to proliferation of various cells, including neoplastic cells, and promotes growth (van Kruijsdijk et al., 2009). Thus, it has been proposed that hyperproduction of leptin may contribute to tumor development. In the present study, serum leptin concentrations of male mice fed HFD for 13 and 26 weeks were 9.3 and 3.8 times higher, respectively, than those of mice fed STD for the same time periods. Among female mice, serum leptin concentrations in HFD-fed mice were 4.3 and 9.7 times higher than those of mice fed STD for the same time periods. These findings indicate that the degree of obesity under the present experimental conditions was sufficient for investigation of the effects of obesity on spontaneous gene mutations. The tissues examined in the present study, liver, kidney, and colon, were selected because they have been proposed to be associated with obesity-related cancer development on the basis of epidemiological studies (Renehan et al., 2008; World Cancer Research Fund, 2007). Thus, the organs examined in present study were considered to be reasonable target tissues in which to investigate the effects of obesity on the spontaneous occurrence of somatic gene mutations.

To investigate the effects of obesity on spontaneous gene mutations, we performed reporter gene mutation assays of liver, kidney, and colon from mice, and demonstrated that there were no significant differences between the STD- and HFD-fed groups. Our previous data demonstrated that the spontaneous gpt MFs in the colon of male C57BL/6 gpt delta mice at 11 weeks of age was $0.82 \pm 0.43$ (Means $\pm S D, n=5$ ) (Okamura et al., 2010). The values at 19 and 32 weeks of age in the present study were $0.38 \pm 0.12$ and $0.95 \pm 0.64$, respectively. Therefore, the values of gpt MFs in the present study seem to be within the same range of data from the previous study. Thus, the present data showing no significant increase in spontaneous gene mutations in the organs associated with obesity-related cancer even under conditions of severe obesity suggest that HFD treatment and consequent obesity do not affect the frequency of spontaneous somatic gene mutations in spite of the condition being effective on the tumor promotion.

In conclusion, we demonstrated that obesity induced by HFD did not influence the frequency of reporter gene mutations in liver, kidney, or colon from gpt delta mice. Therefore, the tumor-promoting effects of obesity may not involve spontaneous gene mutations, suggesting that obesity may contribute only to the promotion of carcinogenesis.

\section{Acknowledgements}

This work was supported by a Grant-in Aid for Research on Food Sanitation from the Ministry of Health, Labor and Welfare of Japan.

\section{References}

Aoki Y, Hashimoto AH, Amanuma K, et al (2007). Enhanced spontaneous and benzo(a)pyrene-induced mutations in the lung of Nrf2-deficient gpt delta mice. Cancer Res, 67, 5643-8.

Bjorbaek C, Kahn BB (2004). Leptin signaling in the central nervous system and the periphery. Recent Prog Horm Res, 59, 305-31.

Ceddia RB (2005). Direct metabolic regulation in skeletal muscle and fat tissue by leptin: implications for glucose and fatty acids homeostasis. Int J Obes (Lond), 29, 1175-83.

Hirose Y, Hata K, Kuno T, et al (2004). Enhancement of development of azoxymethane-induced colonic premalignant lesions in C57BL/KsJ-db/db mice. Carcinogenesis, 25, $821-5$.

Iwasa J, Shimizu M, Shiraki M, et al (2010). Dietary supplementation with branched-chain amino acids suppresses diethylnitrosamine-induced liver tumorigenesis in obese and diabetic C57BL/KsJ-db/db mice. Cancer Sci, 101, 460-7.

Lee GH, Proenca R, Montez JM, et al (1996). Abnormal splicing of the leptin receptor in diabetic mice. Nature, 379, 632-5.

Masumura K, Sakamoto Y, Ikeda M, et al (2011). Antigenotoxic effects of p53 on spontaneous and ultraviolet light B-induced deletions in the epidermis of $g p t$ delta transgenic mice. Environ Mol Mutagen, 52, 244-52.

Morrison DS, Parr CL, Lam TH, et al (2013). Behavioural and metabolic risk factors for mortality from colon and rectum cancer: analysis of data from the Asia-Pacific Cohort Studies Collaboration. Asian Pac J Cancer Prev, 14, 1083-7.

Nohmi T, Suzuki T, and K. Masumura K (2000). Recent advances in the protocols of transgenic mouse mutation assays. Mutat Res, 455, 191-215.

Okamura T, Ishii Y, Suzuki Y, et al (2010). Effects of co-treatment of dextran sulfate sodium and MeIQx on genotoxicity and possible carcinogenicity in the colon of p53-deficient mice. J Toxicol Sci, 35, 731-41.

Padidar S, Farquharson AJ, Williams LM, et al (2012). High-fat diet alters gene expression in the liver and colon: links to increased development of aberrant crypt foci. Dig Dis Sci, 57, 1866-74.

Reddy BS, Maeura Y (1984). Tumor promotion by dietary fat in azoxymethane induced colon carcinogenesis in female F344 rats: influence of amount and sources of dietary fat. $J$ Natl Cancer Inst, 72, 745-50.

Renehan AG, Tyson M, Egger M, Heller RF, Zwahlen M (2008). Body-mass index and incidence of cancer: a systematic review and meta-analysis of prospective observational studies. Lancet, 371, 569-78.

Shimizu M, Sakai H, Shirakami Y, et al (2011). Preventive effects of (-)-epigallocatechin gallate on diethylnitrosamine-induced liver tumorigenesis in obese and diabetic C57BL/KsJ- $d b / d b$ Mice. Cancer Prev Res, 4, 396-403.

Trak-Smayra V, Paradis V, Massart J, et al (2011). Pathology of the liver in obese and diabetic $o b / o b$ and $d b / d b$ mice fed a standard or high-calorie diet. Int J Exp Pathol, 92, 413-21.

van Kruijsdijk RC, van der Wall E, Visseren FL (2009). Obesity and Cancer: The role of dysfunctional adipose tissue. Cancer Epidemiol Biomarkers Prev, 18, 2569-78.

Vansaun MN (2013). Molecular pathways: adiponectin and leptin signaling in cancer. Clin Cancer Res, 19, 1926-32.

World Cancer Research Fund, / American Institute for Cancer Research (2007). Food, Nutrition, Physical Activity, and the Prevention of Cancer: a Global Perspective. Washington DC: AICR. 